\title{
General Proof of Osterwalder-Schrader Positivity for the Wilson Action
}

\author{
P. Menotti ${ }^{1}$ and A. Pelissetto ${ }^{2}$ \\ ${ }^{1}$ Dipartimento di Fisica dell'Università di Pisa, and INFN, Sezione di Pisa, I-56100 Pisa, Italy \\ ${ }^{2}$ Scuola Normale Superiore, Pisa, and INFN, Sezione di Pisa I-56100 Pisa, Italy
}

\begin{abstract}
We extend the proof by Osterwalder and Seiler of reflection positivity of lattice gauge theories with fermions to the case in which the reflection is performed with respect to planes containing sites. Our proof applies to all observables and relies on the particular structure of the Wilson action. This assures the positivity of the correlation functions $\langle\theta(F) F\rangle$ for any gauge invariant function $F$ and at all euclidean time separations as of interest in Monte Carlo calculations.
\end{abstract}

\section{Introduction}

A problem in lattice gauge theories [1] is that of proving the OsterwalderSchrader [2] positivity condition which is one of the fundamental ingredients for reconstructing Wightman theory from euclidean theory. From the practical viewpoint the positivity condition excludes the existence of ghosts from the physical sector of the theory.

Lüscher [3] and independently Osterwalder and Seiler [4] have addressed the problem in two different ways. Lüscher starting from the Wilson action builds up a Hilbert space as a Fock space derived from equal time fields and explicitly constructs a transfer matrix [5] which he proves to be positive definite. In this context Osterwalder-Schrader positivity is proved for gauge invariant operators constructed from the fields belonging to given time slices. Osterwalder and Seiler on the other hand prove the Osterwalder-Schrader positivity condition directly from the functional integral for all gauge invariant quantities. Their procedure is sufficient to construct a positive hamiltonian on a Hilbert space; however they prove the positivity of $\langle\theta(F) F\rangle$ only by reflecting with respect to a plane cutting the time links in half (i.e. for "odd" separations). This situation is not completely satisfactory for the Monte-Carlo calculations where one computes correlation functions of arbitrary gauge invariant composite operators at all (even and odd) distances.

In this note we shall prove the Osterwalder-Schrader positivity condition for all gauge invariant quantities for any separation in the case of the Wilson action. 
The following proof does not depend on the explicit construction of a Hilbert space as a Fock space and on operators defined on time slices (sharing in this respect the advantages of the Osterwalder-Seiler treatment) and on the other hand it applies for any separation (sharing in this respect the advantages of the Lüscher construction).

The following general proof turns out to be very simple even though (as in Lüscher's case) it applies only to the Wilson action.

\section{Proof of Osterwalder-Schrader Positivity}

In this section we shall give the general proof of reflection positivity for the lattice gauge theory with fermions described by the Wilson action

$$
A=A_{G}+A_{F},
$$

where

$$
A_{G}=\frac{1}{2 g_{0}^{2}} \sum_{\substack{\mu \neq v \\ n}} \operatorname{Tr}\left\{U_{\mu}(n) U_{v}(n+\hat{\mu}) U_{\mu}^{+}(n+\hat{v}) U_{\nu}^{+}(n)\right\}
$$

and

$$
\begin{aligned}
A_{F}= & \sum_{n}\left\{-\bar{\psi}(n) \psi(n)+K \sum_{\mu=1}^{4}\left[\bar{\psi}(n)\left(1+\gamma_{\mu}\right) U_{\mu}(n) \psi(n+\hat{\mu})\right.\right. \\
& \left.\left.+\bar{\psi}(n+\hat{\mu})\left(1-\gamma_{\mu}\right) U_{\mu}^{+}(n) \psi(n)\right]\right\} .
\end{aligned}
$$

We shall prove that, provided $K<1 / 6$ [3], given any gauge invariant function $F$ of $\psi, \bar{\psi}$ and $U$ belonging to a certain region $R$ of the half-space $n_{4}>0$, the correlation function of $\theta(F)$ and $F$ is always non-negative, i.e.

$$
Z^{-1} \int d U d \bar{\psi} d \psi e^{A}(\theta F) F \equiv\langle(\theta F) F\rangle \geqq 0
$$

being $\theta(F)$, the Osterwalder-Schrader reflection of $F$ defined as follows $[2,4]$ (we shall write $U_{\mu}(n)$ as $\left.U(n, n+\hat{\mu})\right)$ :

$$
\begin{aligned}
\theta\left(\vec{n}, n_{4}\right) & =\left(\vec{n},-n_{4}\right), \\
\theta(U(n, n+\hat{\mu})) & =U(\theta n, \theta(n+\hat{\mu})), \\
\theta[G(U)] & =G^{*}(\theta U), \\
\theta[\psi(n)] & =\bar{\psi}(\theta n) \gamma_{4}, \\
\theta[\bar{\psi}(n)] & =\gamma_{4} \psi(\theta n),
\end{aligned}
$$

and for a monomial of the $\psi, \bar{\psi}$

$$
\begin{aligned}
& \theta\left[f(U) \psi\left(n_{1}\right) \ldots \bar{\psi}\left(n_{k}\right) \ldots\right] \\
& \quad=f^{*}(\theta U)\left(\ldots \theta\left[\bar{\psi}\left(n_{k}\right)\right] \ldots \theta\left[\psi\left(n_{1}\right)\right]\right),
\end{aligned}
$$

where $G^{*}$ and $f^{*}$ is the usual complex conjugation on $c$-numbers. The reflection operator $\theta$ is extended by antilinearity to an arbitrary polynomial of the $\psi, \bar{\psi}$. In 
order to have a positive definite transfer matrix it is necessary to prove such a positivity property by reflecting both with respect to planes cutting the time links in half and with respect to planes containing sites.

Osterwalder and Seiler [4] gave the general proof of (2.4) for reflection with respect to planes cutting the time links in half. Essentially their procedure is to choose the gauge $U_{4}(n)=1$ (it is enough to have this gauge on the links cut by the reflecting plane) and noticing that the action can be written in the form

$$
A=A^{+}+\theta A^{+}+A_{C},
$$

where $A_{C}$ can be decomposed in the sum $A_{C G}+A_{C F}$ with

$$
\begin{aligned}
A_{C G} & =\frac{1}{2 g_{0}^{2}} \sum_{\vec{n}, \ell} \operatorname{tr}\left(U_{\ell}\left(\vec{n}, \frac{1}{2}\right) U_{\ell}^{+}\left(\vec{n},-\frac{1}{2}\right)\right)+\text { c.c. } \\
& =\frac{1}{2 g_{0}^{2}} \sum_{\vec{n}, \ell} U_{\ell}^{i j}\left(\vec{n}, \frac{1}{2}\right) \theta\left[U_{\ell}^{i j}\left(\vec{n}, \frac{1}{2}\right)\right]+\text { c.c. }
\end{aligned}
$$

and

$$
\begin{aligned}
A_{C F}= & K \sum_{\vec{n}}\left\{\bar{\psi}\left(\vec{n},-\frac{1}{2}\right)\left(1+\gamma_{4}\right) \psi\left(\vec{n}, \frac{1}{2}\right)\right. \\
& \left.+\bar{\psi}\left(\vec{n}, \frac{1}{2}\right)\left(1-\gamma_{4}\right) \psi\left(\vec{n},-\frac{1}{2}\right)\right\} \\
= & K \sum_{\vec{n}}\left\{\theta\left[\psi\left(\vec{n}, \frac{1}{2}\right)\right]\left(1+\gamma_{4}\right) \psi\left(\vec{n}, \frac{1}{2}\right)\right. \\
& \left.+\theta\left[\bar{\psi}\left(\vec{n}, \frac{1}{2}\right)\right]\left(1-\gamma_{4}\right) \bar{\psi}\left(\vec{n}, \frac{1}{2}\right)\right\} .
\end{aligned}
$$

Thus the expectation value (2.4) can be written as a sum of the form [4]

$$
\sum_{i}\left|\int d U d \bar{\psi} d \psi \mathscr{F}_{i}(U, \psi, \bar{\psi})\right|^{2} \geqq 0,
$$

where $U, \psi$ and $\bar{\psi}$ are the fields in the upper half space $n_{4}>0$.

As noticed by Osterwalder and Seiler [4] such a proof does not depend on the choice of the Wilson action and in particular it holds when the projectors $\left(1+\gamma_{4}\right)$ and $\left(1-\gamma_{4}\right)$ in $(2.3)$ are replaced by $\left(r+\gamma_{4}\right),\left(r-\gamma_{4}\right), 0 \leqq r \leqq 1$ which includes as a particular case $(r=0)$ the doubled fermion case.

We come now to the proof of (2.4) for reflections with respect to the plane containing sites $n_{4}=0$. In the following we shall understand for variables belonging to the upper half space $V_{4}^{+}$all $\psi(n), \bar{\psi}(n)$ with $n_{4}>0$ and all $U(n, m)$ with either $n_{4}>0$ or $m_{4}>0$. A similar definition holds for the variables belonging to the half space $V_{4}^{-}$, while for the variables belonging to the 3-dimensional space $V_{3}^{0}$, we mean all $\psi(n), \bar{\psi}(n)$ with $n_{4}=0$ and all $U(n, m)$ with both $n_{4}=0$ and $m_{4}=0$. Accordingly we shall decompose the action $A_{G}$ as the sum

$$
A_{G}=A_{G}^{+}+A_{G}^{-}+A_{G}^{0},
$$

where $A_{G}^{0}$ depends only on the variables belonging to $V_{3}^{0}, A_{G}^{+}$only on the variables belonging to $V_{4}^{+}$and $V_{3}^{0}$, and $A_{G}^{-}$only on the variables belonging to $V_{4}^{-}$and $V_{3}^{0}$.

We write a similar decomposition for the fermion action

$$
A_{F}=A_{F}^{+}+A_{F}^{-}+A_{F}^{0} \text {. }
$$


In particular

$$
\begin{aligned}
A_{F}^{0}= & -\sum_{\vec{n}} \bar{\psi}(\vec{n}, 0) \psi(\vec{n}, 0) \\
& +K \sum_{\vec{n}} \sum_{j=1}^{3}\left\{\bar{\psi}(\vec{n}, 0)\left(1+\gamma_{j}\right) U_{j}(\vec{n}, 0) \psi(\vec{n}+\hat{j}, 0)\right. \\
& \left.+\bar{\psi}(\vec{n}+\hat{j}, 0)\left(1-\gamma_{j}\right) U_{j}^{+}(\vec{n}, 0) \psi(\vec{n}, 0)\right\} .
\end{aligned}
$$

At this point we must discuss the dependence of $A_{F}^{+}$and $A_{F}^{-}$on the Fermi variables belonging to the plane $n_{4}=0$. The terms in the fermion lagrangian which connect two different time slices are

$$
K\left\{\bar{\psi}(n)\left(1+\gamma_{4}\right) U_{4}(n) \psi(n+\hat{4})+\bar{\psi}(n+\hat{4})\left(1-\gamma_{4}\right) U_{4}^{+}(n) \psi(n)\right\},
$$

which following Lüscher's notations [3] in terms of half spinors

$$
\begin{gathered}
\psi=\left(\begin{array}{c}
x \\
y^{+}
\end{array}\right), \quad \bar{\psi}=\left(\begin{array}{c}
x^{+} \\
-y
\end{array}\right), \\
\gamma_{j}=i\left(\begin{array}{cc}
0 & \sigma_{j} \\
-\sigma_{j} & 0
\end{array}\right), \quad \gamma_{4}=\left(\begin{array}{rr}
1 & 0 \\
0 & -1
\end{array}\right),
\end{gathered}
$$

becomes

$$
K\left\{x^{+}(n) U_{4}(n) x(n+\hat{4})-y(n+\hat{4}) U_{4}^{+}(n) y^{+}(n)\right\} .
$$

Thus we have

$$
A_{F}^{+}=\mathscr{S}\left(U, \psi, \bar{\psi}, x^{+}(\vec{n}, 0), y^{+}(\vec{n}, 0)\right)
$$

with $U, \psi, \bar{\psi}$ belonging to $V_{4}^{+}$, and

$$
A_{F}^{-}=\tilde{\mathscr{P}} *(\theta U, \theta \psi, \theta \bar{\psi}, x(\vec{n}, 0), y(\vec{n}, 0)),
$$

where * means the usual complex conjugation on $c$-numbers and ${ }^{\sim}$ means that the order of the Fermi fields has to be reversed.

Integrating on all fermion and $U$ variables belonging to $V_{4}^{+}$and to $V_{4}^{-}$, we get for the expectation value (2.4),

$$
\begin{aligned}
& Z^{-1} \int \prod_{\vec{n}, j} d U_{j}(\vec{n}, 0) d \bar{\psi}(\vec{n}, 0) d \psi(\vec{n}, 0) e^{A_{G}^{0}+A_{T}^{0}} \\
& \quad \times \tilde{J}^{*}(U(\vec{n}, 0), x(\vec{n}, 0), y(\vec{n}, 0)) \\
& \quad \times \mathscr{J}\left(U(\vec{n}, 0), x^{+}(\vec{n}, 0), y^{+}(\vec{n}, 0)\right) .
\end{aligned}
$$

On the other hand action $A_{F}^{0}$ can be written as

with

$$
A_{F}^{0}=A_{1}+A_{2}+A_{3},
$$

where [3]

$$
\begin{gathered}
A_{1}=-x^{+} B x+y B y^{+}, \\
A_{2}=x^{+} c y^{+}, \quad A_{3}=y c^{+} x,
\end{gathered}
$$

$$
\begin{aligned}
B_{\vec{m} . \vec{n}}^{\alpha \beta}= & \delta_{\vec{m}, \vec{n}} \delta^{\alpha \beta} \\
& -K \sum_{j=1}^{3}\left(\delta_{\vec{m}+\hat{j} \cdot \vec{n}} U_{j}^{\alpha \beta}(\vec{m}, 0)+\delta_{\vec{m}, \vec{n}+\hat{j}} U_{j}^{+\alpha \beta}(\vec{n}, 0)\right)
\end{aligned}
$$


being $\alpha, \beta$ color indices and

$$
\begin{aligned}
c_{\vec{m}, \vec{n}}^{\alpha \beta}= & K \sum_{j=1}^{3}\left(\delta_{\vec{m}+\hat{j}, \vec{n}} i \sigma_{j} U_{j}^{\alpha \beta}(\vec{m}, 0)\right. \\
& \left.-\delta_{\vec{m}, \vec{n}+\hat{j}} i \sigma_{j} U_{j}^{+\alpha \beta}(\vec{n}, 0)\right) .
\end{aligned}
$$

$B$ is easily seen to be positive definite [3] for $K<1 / 6$.

Thus we have for the fermionic integration in (2.19)

$$
\begin{aligned}
& \int \prod d x^{+}(\vec{n}, 0) d x(\vec{n}, 0) d y^{+}(\vec{n}, 0) d y(\vec{n}, 0) \\
& \times e^{-x^{+} B x-y^{+} B^{T} y} e^{x^{+} c y^{+}+y c^{+} x} \\
& \times \tilde{J}^{*}(U, x, y) \mathscr{J}\left(U, x^{+}, y^{+}\right) \equiv P(U) .
\end{aligned}
$$

Due to the positivity of $B, x^{+} B x=x^{+} B^{1 / 2} B^{1 / 2} x$, and thus $P(U) \geqq 0$. Finally integrating in $d U$ we have

$$
Z^{-1} \int \prod_{\vec{n}, j} d U_{j}(\vec{n}, 0) e^{A} \stackrel{0}{G} P(U) \geqq 0 .
$$

We stress that the proof succeeds due to the separation of the $x^{+}, y^{+}$variables form the $x, y$ in $\mathscr{J}$ and $\tilde{J}^{*}$ which gives (2.24) the structure of a scalar product in the Grassmann variables. Again this is a consequence of the projectors $\left(1+\gamma_{4}\right)$ and $\left(1-\gamma_{4}\right)$ appearing in the Wilson action. Defining now the transfer matrix $T$ as the transition of a single lattice spacing in the positive euclidean time direction, one proves with a standard procedure $[4]$ that $0 \leqq T \leqq 1$.

Acknowledgement. We are grateful to S. Caracciolo for useful discussions.

\section{References}

1. Wilson, K.G.: Phys. Rev. D 10, 2445 (1974); Quarks and strings on a lattice. In: New Phenomena in subnuclear physics. A. Zichichi (ed.) New York: Plenum 1977;

Polyakov, A.M.: Phys. Lett. 59 B, 82 (1975);

Seiler, E.; Gauge theories as a problem of constructive quantum field theory and statistical mechanics. Lecture Notes in Physics, Vol. 159. Berlin, Heidelberg, New York: Springer 1982

2. Osterwalder, K., Schrader, R.: Commun. Math. Phys. 31, 83 (1973), 42, 281 (1975);

Osterwalder, K.: Euclidean Green's functions and Wightman distributions. In: Constructive quantum field theory. Velo, G., Wightman, A.S. (eds.). Proceedings of the 1973 Erice Summer School. Lecture Notes in Physics, Vol. 25. Berlin, Heidelberg, New York: Springer 1973

3. Lüscher, M.: Commun. Math. Phys. 54, 283 (1977)

4. Osterwalder, K., Seiler, E.: Ann. Phys. (NY) 110, 440 (1978); see also Seiler, R.: ref. 1

5. See also Creutz, M.: Phys. Rev. D 15, 1128 (1977) and "Species doubling and transfer matrices for fermionic fields," preprint BNL-38765

Communicated by K. Osterwalder 
